\title{
Location Control Techniques of Object via Mapping Gesture on Touch Screen to 3-Dimensional Coordinates
}

\author{
Bongen $\mathrm{Gu}^{1}$ and Yoonsik Kwak ${ }^{2 *}$ \\ Dept. of Computer Engineering, Korea National University of Transportation, \\ 1'bggoo@ut.ac.kr, ²yskwak@ut.ac.kr
}

\begin{abstract}
Mobile devices are very popular electronic tools in modern life. Therefore researchers develop new services based on mobile device. The many fields of these services support 3dimensional environment to enhance the feeling of reality. However input facilities equipped in mobile device have constraints to control the location of objects in space simulated by 2dimensional screen. In this paper, we propose new input techniques to control the location of object in 3-dimensional space. Our method traces user's touch and move action on touch screen, decodes actions, and translates into 3-dimensional coordinates. To trace user's touch and move action, we use one or two fingers gestures. We implement sample Android application adopting our techniques. From our experience of using sample implementations, we can know that our input method can be effectively used to control the location of object in 3-dimensional space.
\end{abstract}

Keywords: gesture, one finger gesture, two fingers gesture, 3-d coordinates, mobile, input

\section{Introduction}

Enhancement of mobile network environment and miniaturization of IT devices make mobile devices popular. In this mobile environment, it is possible to develop new mobile services such as game, virtual reality, and augmented reality, etc. To increase the feeling of reality and immersion, these services provide contents based on 3-dimensional environment [1], and many researchers develop new user interfaces [2]. Among studies on user interface, a touch-based hand gesture interface offers new opportunities for human-computer interaction [3]. Especially [3] studied on 2-D gesture interface for human computer interaction. In [3], researcher's approach interpreted asynchronous touches on the surface as conventional singlefinger typing. To resolve the limitation of input on small touch screen, some researchers used IR(Infra-Red) proximity sensor [4]. In [4], they presented a prototype device with IR proximity sensors embedded along each side and capable of detecting the presence and position of fingers.

In the case of mobile game, 3-dimensional game providing the 3-dimensional point of view in the gaming environment is relatively more attractive than 2-dimensional game. Especially, services such as modeling building and high-resolution 3-dimensional game require highlevel virtual reality to provide the enhanced feeling of reality.

To provide services based on 3-dimensional space as described above, we need the efficient input method for the location of object located in space. To efficiently input the

\footnotetext{
${ }^{*}$ Corresponding Author
} 
location of object, specific input device is required. However, mobile device has constraints to install or attach such special input device. Input method of mobile device like smart phone is normally small number of hardware buttons and touch screen. Hardware buttons of mobile device are used for turning device on or off and controlling sound volume. As described previously, hardware buttons are used for relatively simple operations. So touch screen is main input method to use mobile device. Contemporary touch screen is plane, and is effective to input 2-dimensional coordinates. And it also supports multi-touch capability. Researches related to multi-touch began until the early of 1980. In [5], researchers had presented how to sense multi-touch and interpolate, etc. However this input method is not suitable to input the location of object located in 3-dimensional space. In [6], researchers introduced 3D positioning techniques for multi-touch display. The first finger is used for direct positioning in the camera plane while the second finger is used for depth positioning [6]. This technique targets CAD application. Therefore this focuses on control of a view of the scene [6].

As described above, input methods for mobile device are touch screen, hardware buttons, and sensors. But the main input method for mobile device is normally touch screen. We can input some actions by touching and moving on screen via finger or pen-shaped touch tool. If there is efficient input method for the location of object in space via 2-dimensional screen, developing applications based on 3-dimensional space is promoted.

In this paper, we propose new techniques to input the location of objects in space by using multi-touch, and experimentally implement simple application adopting our techniques. Our method does not require the additional cost, and reduce the mobility to use application based on 3-dimensional space because special input device need not be installed or attached to mobile device.

\section{Motivation}

Currently there are many mobile applications based on 3-dimensional space. Nitendo 3DS titles and smart phone games are examples of these applications. Nintendo 3DS is popular mobile game console, and has many big fans. This console basically supports 3-dimensional game title, and especially can controls the feeling depth of space. And this has special screen to display 3-dimensional game. So users need not to wear special device like polarized glasses to enjoy game title.

Currently the number of smart phone users dramatically increases. So development of game title based on smart phone is stimulated. To increase the reality of game, many 3dimensional game titles are developed and distributed even though smart phone has hardware constraints.

The background of many 3-dimensional mobile games represents 3-dimensional scene. However the movement of characters or objects is not in space. It means that direction of movement is on 2-dimensional plane like horizontal and vertical, or horizontal and depth, or vertical and depth axes. In other words, it displays 2-dimensional movement on 3dimensional background. In summary, the constraints of 3-dimensional services on mobile device are the followings:

- Constraints of object movement

- Absence of input method to control the movement of object

In this paper, we focus on the input method to control the movement of object in space. As described above, mobile device installs 2-dimensional touch screen as primary input facility. We can input for some operations via touch screen by touching and moving finger or tools on 
the surface of screen. So we can use and control mobile device by doing these actions such as touch and moving.

Nintendo 3DS has the slide pad and hardware buttons to control game [7]. The slide pad is touch-based 2-dimensional pad, and is equipped in the bottom piece of game console. Gamer can control the 2-dimensional movement of object via these input facilities even though the background is 3-dimensional scene. The reason is that the input facilities such as the slide pad and 4-direction buttons equipped in this console is basically 2-dimension oriented devices. So gamer has the constraints for controlling object's movement even though game console supports 3-dimensional display.

\section{Mapping One Finger Gesture To Coordinates in Space}

In this paper, we propose a input method for 3-dimensional coordinates by using touch screen which is the primary input facility of mobile device. Our input method traces user's touch and move actions on touch screen, and decodes actions to generate 3-dimensional coordinates. The acquired coordinate via touch screen/pad is 2-dimensional because a contemporary touch screen or pad is based on 2-dimensional plane. Therefore, the coordinate system for touch screen/pad uses two axes: $\mathrm{x}$ - and $\mathrm{y}$-axis. Normally, the $\mathrm{x}$-axis is parallel to horizontal axis of touch screen/pad equipped on mobile device. The y-axis is parallel to vertical axis of touch screen/pad. The touch and move action is defined as followings: First, we contact the surface of screen with finger or pen-shaped tool. And then, we move finger or tool on screen still contacting. This action generates series of 2-dimensional coordinates.

Our input method proposed in this paper analyzes these series of 2-dimensional coordinates, and translates these to 3-dimensional coordinates. The 3-dimensional coordinate system uses three axes orthogonally crossed on the origin to represent the location of object in space. Three axes are $\mathrm{x}-, \mathrm{y}$-, and $\mathrm{z}$-axis. The coordinate in this system represents the location of object in space corresponding to each axis, and consists of three coordinates for each axis. To use touch screen for entering the location of object in space, our method 3-dimensional coordinate from series of 2-dimensional coordinate generated from user's touch and move action.

In our method, we classify user's touch and move action into three directions: horizontal, vertical, and diagonal direction. The horizontal and vertical directions are parallel move with the horizontal and vertical direction of touch screen respectively. The diagonal direction is parallel move with the diagonal line from the lower left corner to the upper right corner on screen. In our method, three classification of user's move action is decoded to 3-dimensional coordinates in space. In other words, our input method translates user's horizontal, vertical, diagonal move directions into $\mathrm{x}-, \mathrm{y}-$-, $\mathrm{z}$-coordinates respectively.

Figure 1 shows the state transition of our technique. Analyzing gesture starts when a finger touches down on a screen. The movement of finger triggers the coordinates changes according to the movement direction. From the diagram shown in figure 1, we know that this one finger gesture can control coordinates of just one axis per movement. 


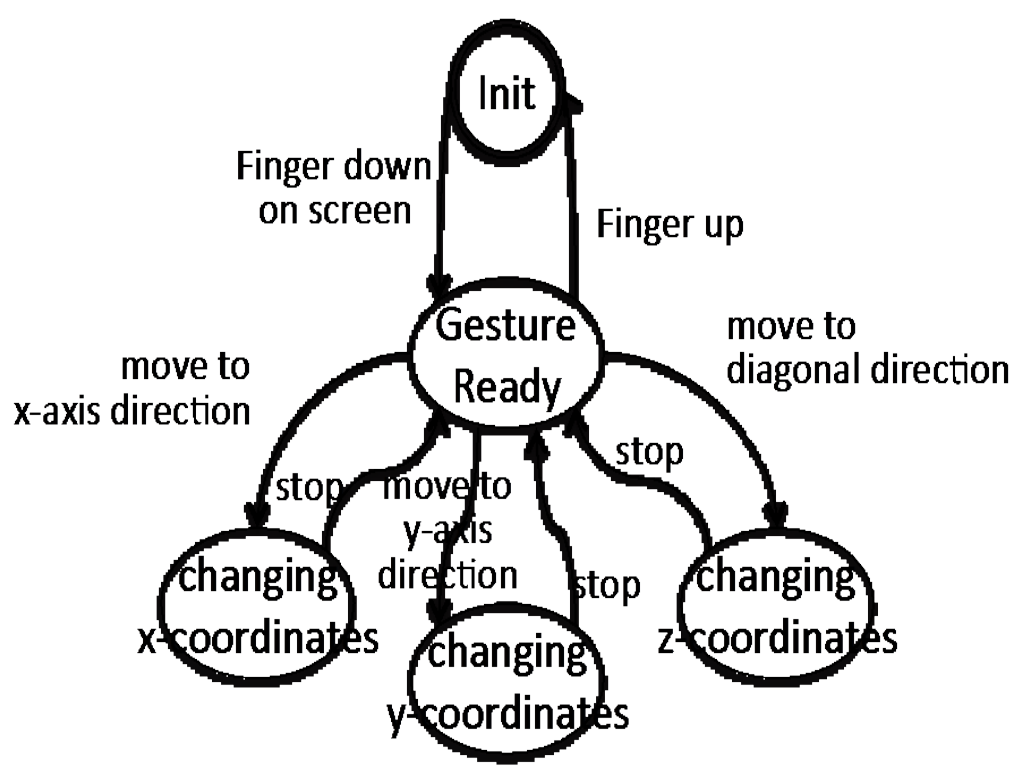

Figure 1. State Transition Diagram Representing the Single Finger Gesture Technique

To derive and solve the problem arisen from using our method to input the location of objects in space, we implement very simple application adopted by our method on mobile device. Our target mobile platform is Android announced by Google, Inc. This platform is very popular, and many mobile device vendors support Android.

The size of human fingers and the lack of sensing precision can make precise touch screen interaction difficult [5]. However the sensing precision was improved by many techniques and researches. While new techniques improve the precision of sensing, the problem for implementing our input method is the following: user's move direction is not completely parallel to the axis. Figure 2 shows the case described above. In this case shown in figure 2, it does not clearly determine whether user's move action is classified into horizontal or diagonal direction. If user's action shown in figure 2 is in the horizontal direction class, this action means the change of x-coordinate in space. On the contrary, if this is in the diagonal direction class, this can change the location of object over z-axis.

We resolve the problem by using the direction angle tolerance. The definition of the direction angle tolerance is the angle range between user's move direction and a axis, so two direction is decided to be parallel. In other words, if the angle between user's move direction and some axis is lower than the angle tolerance, user's move direction is in the axis class. In the example shown in figure 2, if the angle between user's move direction and horizontal direction of screen is lower than the predetermined angle tolerance, our input method can classify user's move direction into horizontal direction class. So the location of sphere in this example is changed over $\mathrm{x}$-axis. 


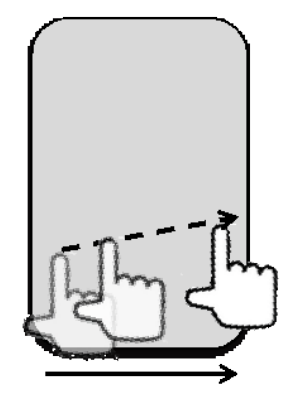

Figure 2. In the Case that User's Move Direction in not Completely Parallel with Horizontal Edge of Mobile Device Screen

\section{Mapping Two Fingers Gesture To Coordinates in Space}

One finger gesture on the touch screen can just control coordinates of one axis per move action. This input method is suitable to control coordinates of just one axis in detail. But it is not suitable to applications requiring complex location control of objects in space, namely, if we should control location of objects in space just with one direction, it lets the feeling of reality and immersion fall off. 3D games and virtual reality applications are in this class. We try to resolve this problem by controlling coordinates of two or more axes concurrently.

To realize our approach, touch screen equipped on mobile device should support multi touch. Multi-touch is technique sensing two or more contact of fingers with surface of screen. This technique is main input facility of mobile device having limited input device. We think that we can control coordinates of two or more axes by using multi touch technique. To solve the problem of one finger gesture, we propose the input technique for controlling locations of objects in space by controlling coordinates of two axes concurrently.

Our technique uses multi touch to control location of objects in space. In other words, multi touch issued by thumb and index fingers, namely, two fingers gesture controls location of objects. Figures 3 and 4 show two fingers gesture controlling location of objects. Thumb finger of two fingers gesture can move with $\mathrm{x}$-axis or diagonal directions as shown in Figure 3. However it is not comfortable for thumb finger to move with y-axis direction of screen. So our input technique determines $\mathrm{x}$-coordinates and z-coordinates of objects by using thumb finger gesture parallel to x-axis and diagonal direction, respectively. Index finger of two fingers gesture can move with y-axis or diagonal directions as shown in figure 4 . Our technique determines y-coordinates and z-coordinates of objects by using index finger gesture parallel to y-axis and diagonal direction, respectively. As previously described, two fingers gesture can control coordinates of two axes concurrently. Therefore our input technique can contribute to enhance reality of contents displayed by mobile device.

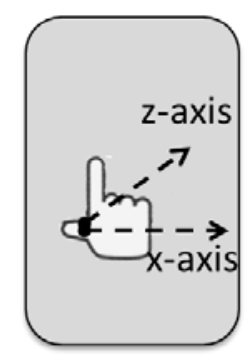

Figure 3. The Movement of Thumb is Parallel to $x$-axis or z-axis 


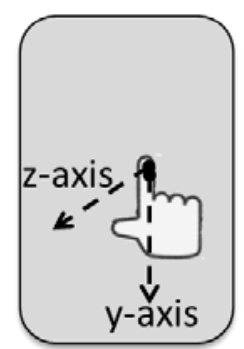

Figure 4. The Movement of Index Finger is Parallel to y-axis or z-axis

To derive and solve the problem arisen from using our two fingers gesture method to control the location of objects in space, we also implement very simple application adopted by our method on mobile device. The problem of implementation adopting our input technique is how to determine thumb and index fingers from two fingers gesture. In our implementation, this problem is solved by recognizing thumb and index finger from leftbottom and right-top touch, respectively, when starting two fingers gesture. By tracking motion of 'thumb' and 'index finger', our technique can change 3-dimensional coordinates of objects. If left-handed person uses our technique, touches for thumb and index fingers are in right-bottom and left-top position when starting gesture. Of course, user should previously configure whether he/she is right-handed or left-handed. In our implementation, we assume that user is right-handed.

The other problem of implementation adopting our technique is how to decide whether movement of two fingers with diagonal direction is for changing $\mathrm{z}$-coordinates or for zooming contents. Our implementation uses this as gesture for zooming contents. Thumb or index finger gesture is exclusively used to change z-coordinates of objects. Therefore two fingers gesture in our technique can change the following coordinates:

- C1: Horizontal movement of the thumb $\rightarrow$ S1: changing $\mathrm{x}$-coordinates

- C2: Diagonal movement of the thumb $\rightarrow$ S2: changing z-coordinates

- C3: Vertical movement of the index finger $\rightarrow$ S3: changing y-coordinates

- C4: Diagonal movement of the index finger $\rightarrow$ S4: changing z-coordinates

- C5: Horizontal and vertical movement of the thumb and index finger, respectively $\rightarrow$ S5: changing $x$ - and $y$-coordinates

- C6: Horizontal and diagonal movement of the thumb and index finger, respectively $\rightarrow$ S6: changing $\mathrm{x}$ - and $\mathrm{z}$-coordinates

- C7: Diagonal and vertical movement of the thumb and index finger, respectively $\rightarrow$ S7: changing $\mathrm{y}$ - and $\mathrm{z}$-coordinates

The capital character ' $C$ ' represents some condition to trigger coordinates change ' $\mathrm{S}$ '. For example, if user moves his/her thumb to horizontal direction (C1), the x-coordinates of object's location becomes changing (S1). 


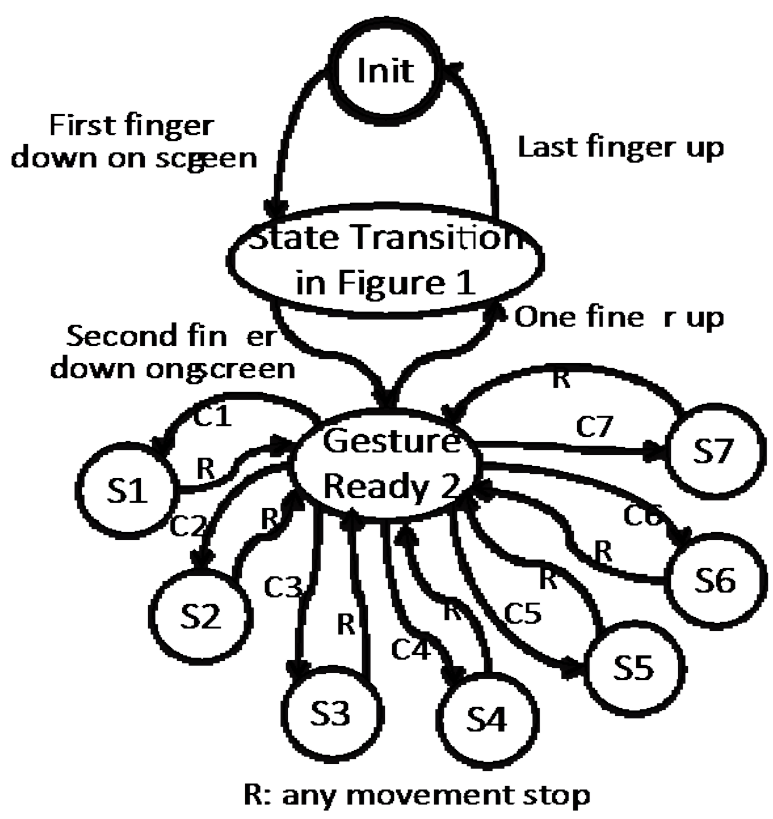

Figure 5. State Transition Diagram Representing the Two Fingers Gesture Technique

Figure 5 shows the state transition of our two fingers gesture technique for controlling the location of object. If the first finger touches on a screen, the state transitions are the same with Figure 1. After the second finger touches on a screen, the state can transition S1 S7 according to the condition, C1 C7.

The problem adopting our technique is that user's move direction is not completely parallel to the axis as previously described. We resolve the problem by using the direction angle tolerance as like one finger gesture above. From our implementations, we can experience the effective location control of objects in space. Therefore we think that our input technique can contribute to give new input facility to mobile device, and to enhance the reality and immersion of contents provided by device of this type.

\section{Conclusion}

Mobile devices are popularized. Therefore many application developers research and make their new services based on mobile device. So the number of mobile services is dramatically increased. Especially, to increase the feeling of the immersion and the reality, 3-dimensional mobile services become popular. To maximize the feeling of space, mobile device should basically support the input method for controlling the location of objects in 3-dimensional space. However, touch screen and hardware button equipped on mobile device have constraints to efficiently control the location of objects. Because these input facilities are basically designed for 2-dimensional plane.

In this paper, we proposed the input method to control the location of object in 3dimensional space by tracing user's touch and move action on 2-dimensional touch screen/pad. Our method decides the coordinate axis from user's move direction, and decodes the coordinate in space. And we implement sample application on Android to adopt our input method. From our experience of using sample application, we think our input method can be used to control the location of object in space efficiently. 


\section{Acknowledgements}

The research was supported by a grant from the Academic Research Program of Korea National University of Transportation in 2013.

\section{References}

[1] H. Iwata, “Artificial reality with force-feedback: development of desktop virtual space with compact master manipulator”, SIGGRAPH '90, New York, USA, (1990) August 06-10.

[2] S. Liawatimena, E. Y. Handy and A. Limas, "User Interface Using Arm Gesture, www.academia.edu/189198/User_Interface_Using_Arm_Gesture.

[3] W. Westerman, J. Elias and A. Hedge, "Multi-Touch: A New Tactile 2-D Gesture Interface for HumanComputer Interaction”, Proceedings of the Human Factiors and Ergonomics Society Annual Meeting, (2001) October.

[4] A. Butler, S. Izadi and S. Hodges, "SideSight: multi-“touch” interaction around small devices”, Proceeding of the 21st annual ACM symposium on User interface software and technology, ACM, New York, USA, (2008).

[5] S. Lee, W. Buxton and K. Smith, “A multi-touch three dimensional touch-sensitive tablet”, Proceedings of the SIGCHI Conference on Human Factors in Computing Systems, ACM, New York, USA, (1985).

[6] A. Martinet, G. Vasiez and L. Grisoni, "The Design and Evaluation of 3D Positioning Techniques for MultiTouch Displays”, 2010 IEEE Symposium on 3D User Interface, Waltham, USA, (2010) March 20-21.

[7] Nintendo, www.nintendo.com.

\section{Authors}

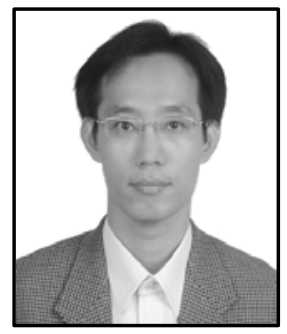

Dr. Bongen Gu, is an Professor of Computer Engineering at Korea National University of Transportation, Korea. He received a Ph.D. degree in Computer Engineering from Kyungpook University, Korea in 1998. His research interests are in the area of HCI, Cloud computing, High Performance Computing, Storage System, High Performance Computer Architecture, and Parallel System.

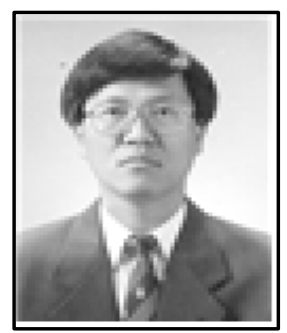

Dr. Yoonsik Kwak, is an Professor of Computer Engineering at Korea National University of Transportation, Korea. He received a Ph.D. degree in Electronic Engineering from Kyunghee University, Korea in 1994.7 His research interests are in the area of Computer vision, Storage system, parallel system, and Internet communication. 